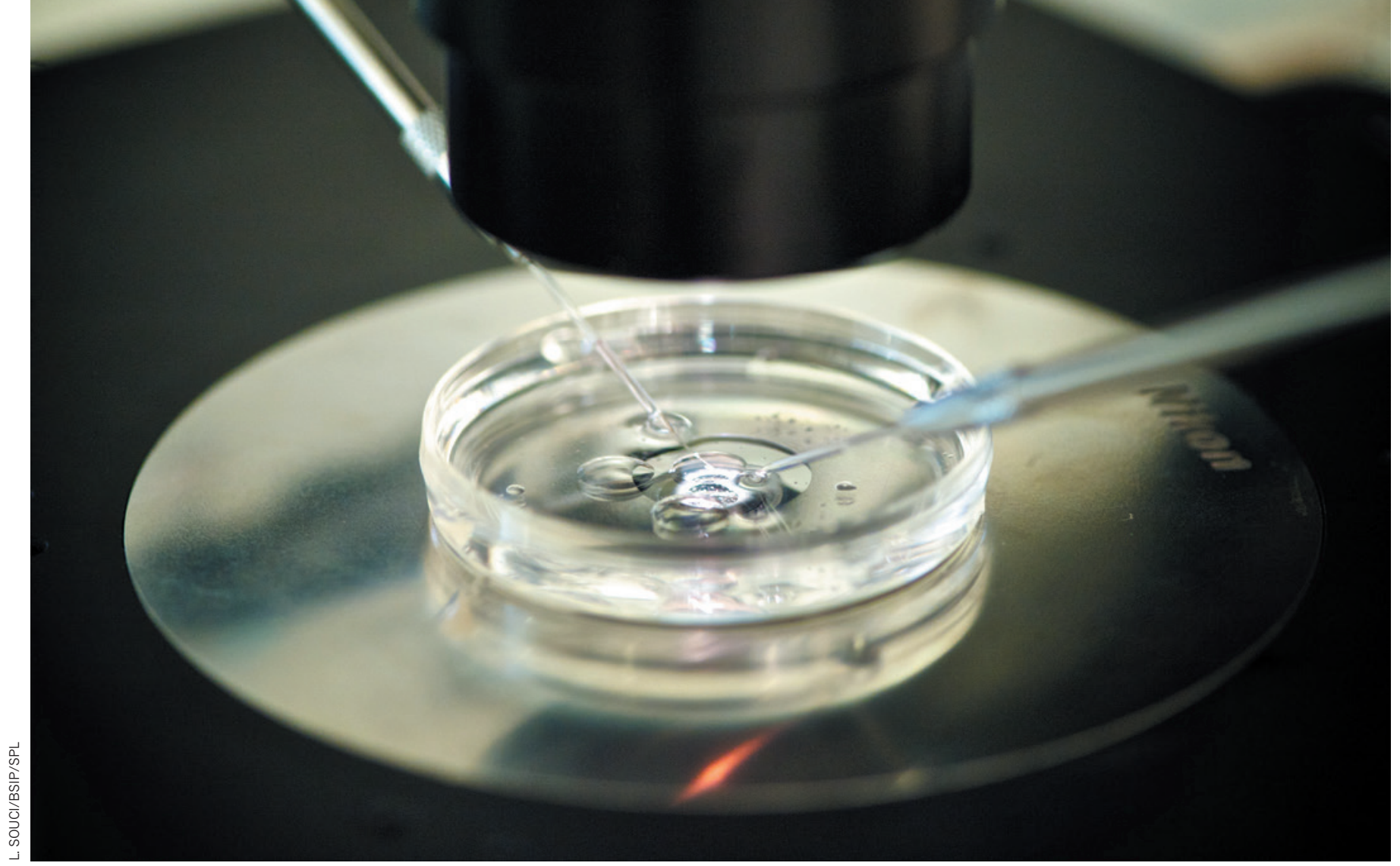

Human embryos are prime targets for genome editing.

\title{
A path through the thicket
}

\section{As various advisory bodies, scientific organizations and funding agencies deliberate on genome editing in humans, Debra J. H. Mathews, Robin Lovell-Badge and colleagues lay out some key points for consideration.}

$\mathrm{T}$ he ease of use, accuracy and efficiency of the genome-editing tool CRISPR/ Cas9 has led to its broad adoption in research, as well as to preliminary applications in agriculture and in gene therapies involving non-reproductive (somatic) cells. It is also possible in some jurisdictions to deploy CRISPR/Cas9, and related techniques ${ }^{1}$, in human germline cells (sperm and eggs) as well as in early embryos ${ }^{2}$.

In September, a network of more than 30 scientists, ethicists, policymakers, journal editors and funders called the Hinxton Group gathered in Manchester, UK, to address the ethical and policy issues surrounding the editing of human genomes in the early stages of development and in germline cells (see go.nature.com/xikxv2). Similar meetings have been and are being held elsewhere in the world, and several position statements have been published (see, for instance, go.nature.com/enfxjz and go.nature.com/ fes1wc). Indeed, the US National Academies of Sciences, Engineering and Medicine is hosting what could be the largest such gathering next month, in concert with the Chinese Academy of Sciences and the Royal

\section{POINTS FOR DEBATE}

Some key questions are emerging as advisory bodies across the world discuss the ethical and policy implications of genome editing in humans. Here are four:

- Should genome editing be allowed in basic research involving human sperm, eggs and embryos?

- Should only embryos left over from in vitro fertilization be used in genomeediting research or may embryos be specifically created for research?

- What safety and efficacy thresholds need to be met before the use of genome editing in human reproductive applications could be considered?

- If such thresholds are met, what uses for genome editing in human reproductive applications might be permissible?
Society in London (see go.nature.com/frauil). Here, we lay out some key points emerging from the Hinxton meeting that are likely to gain more focus from the international community in the coming months. The views presented here are those of the group's steering committee and do not necessarily represent the consensus view of the group.

\section{RECOMMENDED ACTIONS}

Establish a model regulatory framework that could be adopted internationally. Various groups, including ours, agree that numerous technical and safety issues need to be addressed before genome-editing technologies could feasibly be used in reproductive clinical applications. Many also share our strong conviction that basic research involving genome editing should not be halted or hampered. Such studies are likely to have tremendous value, including in humanreproduction applications that do not

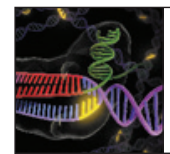

CRISPR GENE EDITING A Nature collection nature.com/crispr 
involve genome editing, and potentially in the development of treatments using somatic cells (see 'Basic benefits').

Even if much of the international scientific community and major funders of biomedical research agree on this point, however, the ease of use and accessibility of the technology make it ripe for exploitation by rogue or charlatan organizations - especially in jurisdictions where fertility clinics, which must be involved, are loosely regulated.

\section{"We could not agree on the merits and downsides of listing possible clinical uses."}

After all, for the

past decade, thousands of medical tourists have collectively paid many millions of dollars to receive unproven and unregulated stemcell interventions internationally ${ }^{3,4}$.

To address this issue, it would be helpful to have a model regulatory framework that is specific enough to be meaningful, but general enough that it could quickly be adopted by country-specific regulatory bodies. There are precedents for such guidance; a document produced by the International Society for Stem Cell Research informs and influences oversight of stem-cell research internationally. But guidelines cannot in themselves prevent clinics from carrying out unproven treatments - that is the remit of local authorities. Much as the Human Fertilisation and Embryology Act, and the regulatory body called the Human Fertilisation and Embryology Authority (HFEA), have set limits around the use of embryos and gametes in research and the clinic in the United Kingdom, clear boundaries need to be established to indicate when research and clinical practice are permitted, with penalties given to those who step outside them. The public must also be made aware of what is legitimate and what is not, which will in turn require robust communication from authorities as well as from scientists and clinicians.

Develop a road map for basic research. Although much of the focus of public discussions has been on potential clinical applications, the immediate and perhaps most exciting uses of human genome editing are in basic research. Advisory and regulatory bodies should set priorities for genome-editing research involving human cells, including the germ line. This should involve canvassing a diverse group of scientists from across the world who have expertise on: genomeediting technologies; genomics and human genetic variation; mutation types, frequency and effects on physical and other traits; gene expression and regulation; epigenetics; human embryology and reproductive biology; and clinical genetics.

Two issues will be particularly crucial to address - both in the context of basic research and especially in relation to the development of any human reproductive applications.

Off-target events. Computer-based and in vitro methods will be required to evaluate the likelihood of introducing changes outside the target site and the possible consequences. They will also be needed to distinguish such mutations from those that result naturally from, for example, imperfect DNA repair after cell division or environmental insults (chemicals, ionizing radiation and so on).

When genome editing is applied to cells that grow well in culture, such as spermatogonial stem cells (which give rise to sperm), a single edited cell can generate many millions of others. By sequencing the genomes of a subset of these cells, researchers can get a good indication of off-target events. Such assays are less reliable when only one or a few cells are available, as with cells biopsied from early embryos. In this case, one option would be to establish pluripotent cell lines from these cells. A key challenge will be to differentiate off-target events from the substantial natural sequence variation that exists between individuals and between cell lines.

Recent experiments ${ }^{5}$ in mice and in human cell lines suggest that the rate of offtarget events is insignificant compared with the number of spontaneous mutations that occur in each generation ${ }^{6}$. Yet the number of mutations may be less important than where they occur. Unlike spontaneous mutations, which are essentially random, off-target events are likely to be influenced by the RNA molecule that is used to guide the cutting component of the technology (the nuclease) to the right place in the genome. Also, most genome-editing research so far has been conducted in genetically homogeneous populations, such as inbred strains of mice. Little is known about what effect variation in genetic background might have on the efficiency and accuracy of genome editing, or on the ability of researchers to differentiate off-target events from background variation.
Mosaicism. When genome-editing tools are applied to multicellular embryos, only some of the cells may be altered, resulting in a genetic mosaic of edited and unedited cells. Even when applied to a single-cell embryo, the nuclease may not cut both copies of the target gene, or the cell may start to divide before the changes have been completed. It will be important to know what level of mosaicism is likely for different applications of genome editing, how this can be measured, and what effects mosaicism might have.

For some research purposes, such as tracking cell fate, mosaicism may not matter. (As long as some of the cells in the original sample carry the marker gene - say, the gene encoding green fluorescent protein - researchers will be able to identify what those cells develop into.) For others, mosaicism could be more problematic. For instance, if the aim is to determine the role of a gene whose product is secreted and the total amount of secreted product is crucial to gene function, then having a proportion of unedited cells will greatly affect the conclusions.

\section{Engage people from all sectors of society in a debate about genome editing, includ- ing the use of human embryos in this research. Although human embryos and sperm and egg cells are used in a broad range of research internationally, including in the context of in vitro fertilization (IVF) and embryonic stem-cell research, some have suggested that their uses in genome-editing research should be considered independently. Importantly, embryos left over from IVF are unlikely to be a good model if mosaicism is to be avoided, because they comprise more than one cell; they are usually not made available for research until they are at or beyond the eight-cell stage of development. Also, founder effects, whereby most of a fetus derives from one or a few early cells, could complicate pre- dictions about the functional consequences of mosaicism. Cell competition could have similar effects. Early analyses may indicate,}

\section{BASIC BENEFITS}

The use of genome editing in human sperm, eggs and embryos could yield valuable insights in several areas of basic research.

\section{Research}

How cell types are specified in the early human embryo, and the nature and importance of the genes involved.

Understanding the biology and genetics of stemcell lines representing the cell lineages thought to exist in the early human embryo - including non-embryonic cells, such as those that go on to make the placenta.

The role of specific genes in human germ-cel development, including the differentiation of sperm and eggs.

Genome-editing techniques.

\section{Possible applications}

Improved techniques for culturing embryos following in vitro fertilization, better implantation rates and fewer miscarriages.

Improved ability to establish stem-cell lines for research, prevent miscarriages and screen drugs for efficacy. Reduction in the need for embryos in research.

Fertility enhancement and the development of new contraceptives.

Improved efficiency and versatility of genome editing in early embryos and in germline cells. Reduction in the numbers of embryos required in experiments. 


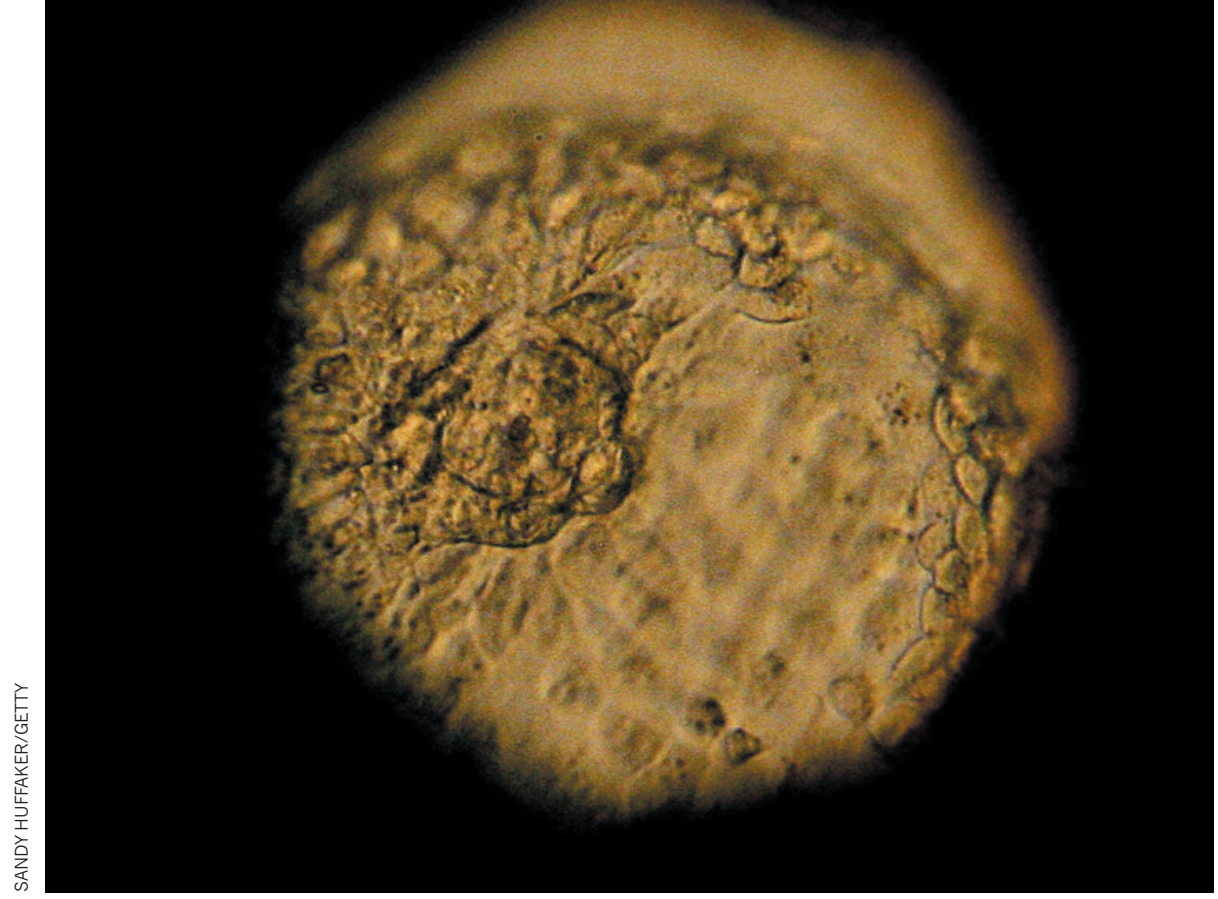

Embryos donated to the La Jolla IVF clinic are made available for stem-cell research in the United States.

for instance, that even if $80 \%$ of the cells in an embryo were manipulated, the resulting fetus could turn out to contain a much smaller proportion of edited cells if such cells differ even very slightly in their rate of division or survival compared with unedited ones ${ }^{7}$.

Minimizing and so reducing the effects of mosaicism could require introducing the genome-editing components - the nuclease and the guide RNA - just after fertilization, or even at the point when fertilization happens ${ }^{8}$. If this is the case, work that involves editing the human germ line may end up being restricted to those jurisdictions that allow the creation of embryos specifically for research. Under current laws, this would limit studies to eight countries: Belgium, China, Israel, Japan, Singapore, South Korea, the United Kingdom and the United States (R. Isasi, personal communication). And in the United States, such work would be possible only with the use of non-federal funds.

If it turns out that major benefits arise from basic research in human-genome editing including applications that do not in themselves involve genome editing, such as ways to improve fertility or reduce the incidence of miscarriage - then national laws could jeopardize people's access to such benefits.

Design tools and methods to enable inclusive and meaningful deliberation. Groups such as the UK Nuffield Council on Bioethics and the national academies in the United States and elsewhere are well placed to take the lead on efforts to ensure that debates over the use of human embryos in basic research are geographically and demographically inclusive, and that such debates inform policy decisions. This is also crucial in relation to the even thornier question of what clinical applications might be appropriate, given sufficient assurance of safety and efficacy.

Even for members of the Hinxton Group - well-informed people who concur that basic research involving genome editing, including that in human sperm, eggs and embryos, has tremendous value - discussions about possible reproductive applications were charged. In fact, we found it impossible to agree on potentially defensible uses for editing the human germ line without having the context and facts of a particular case. The group considered a spectrum of interventions, from the correction of lifethreatening mutations (those that cause TaySachs disease, cystic fibrosis and Huntington's disease, for example), to the introduction of preventive changes (including disruption of the CCR5 gene to confer resistance against HIV infection), to non-medical enhancements (such as increasing muscle mass). Ultimately, we could not even agree on the merits and downsides of listing possible clinical uses - some were concerned that making such a list could be taken as tacit approval.

For decades, people have been arguing about the pros and cons of human germline modification ${ }^{9}$, how to distinguish medical treatments from enhancement, what rights parents have over the lives of their children and so on. Yet good models for how to enable a diversity of perspectives to shape morally contested areas of emerging science and technology are hard to find.

When the US National Institutes of Health (NIH) published draft guidelines for embryonic stem-cell research in 2009, nearly 50,000 people sent in comments. Many of those who weighed in were non-scientists, including about 16,000 who opposed the research on moral grounds. On seeing that their comments had failed to shape the final guidelines, opponents of stem-cell research went to the courts, and four years of litigation and uncertainty for the field followed.

More recently, the HFEA conducted a programme of public engagement that included workshops, focus groups and online surveys to gauge opinion on a range of issues relevant to the use of mitochondrial replacement therapy (MRT) in the clinic. (In MRT, which was approved by the UK government in February, faulty mitochondrial DNA in an egg or embryo is discarded and DNA from a woman without mitochondrial disease is used.) This programme largely achieved its goals and is one of the best examples we have of such an effort (see go.nature.com/64cioj). To some, both of these examples of public engagement constitute successes, but we have no a priori measures to indicate what success means.

Systematic study of various models of engagement is needed to identify, for instance, the best methods for ascertaining not only partisan positions, but also broad societal attitudes. So too are investigations that probe how public deliberations can shape policy.

The advances in genome-editing technologies mean that long-standing ethical questions can no longer be dodged on the basis of obvious and agreed safety concerns. And although continued debate is crucial, it is time to collectively make decisions about the kind of world we want to live in and to develop policies to reflect that vision.

Debra J. H. Mathews is assistant director for science programmes at the Johns Hopkins Berman Institute of Bioethics, Baltimore, Maryland, USA, and associate professor in the Department of Pediatrics at the Johns Hopkins University School of Medicine. Robin LovellBadge is group leader at the Laboratory of Stem Cell Biology and Developmental Genetics, the Francis Crick Institute, London, UK. The rest of the Hinxton Group Steering Committee are Sarah Chan, Peter J. Donovan, Thomas Douglas, Christopher Gyngell, John Harris and Alan Regenberg. e-mail:dmathews@jhu.edu

1. Zetsche, B. et al. Cell 163, 759-771 (2015)

2. Liang, P. et al. Protein Cell 6, 363-372 (2015).

3. Regenberg, A. C., Hutchinson, L. A., Schanker, B. \& Mathews, D. J. H. Stem Cells 27, 2312-2319 (2009).

4. Einsiedel, E. F. \& Adamson, H. Dev. World Bioeth. 12, 35-44 (2012).

5. Tan, E. P., Li, Y., Velasco-Herrera Mdel, C., Yusa, K. \& Bradley, A. Genesis 53, 225-236 (2015).

6. Shendure, J. \& Akey, J. M. Science 349, 14781483 (2015)

7. Sancho, M. \& Rodríguez, T. A. Cell Cycle 13, 9-10 (2014).

8. Suzuki, T., Asami, M. \& Perry, A. C. F. Sci. Rep. 4, 7621 (2014).

9. US President's Commission for the Study of Ethical Problems in Medicine and Biomedical and Behavioral Research. Splicing Life: The Social and Ethical Issues of Genetic Engineering with Human Beings (US Government Printing Office, 1982).

The authors declare competing financial interests. For details, and for full author affiliations, see go.nature.com/bhrwgt. 